\title{
Photonic Approach to Phased Array Application
}

\author{
Eszter UDVARY \\ Dept. of Networked Systems and Services, Budapest University of Technology and Economics, \\ Muegyetem rakpart 3, 1111 Budapest, Hungary \\ udvary.eszter@vik.bme.hu
}

Submitted January 11, 2021 / Accepted June 14, 2021

\begin{abstract}
Many applications, including phased array antennas, require a significant and tunable time delay, which pure electrical methods cannot establish. A variable microwave true-time delay line utilizing a photonic approach is presented in this paper. The variation of the delay is achieved by the application of dispersive fiber and tunable laser. With this method, the tunable delay line can control multiple radiating elements. In this paper, both DWDM and CWDM solutions are theoretically investigated and compared. The experimental work applying DWDM tunable laser validates the theoretical analysis. Finally, the phased array concept is presented, and the system simulation shows the operation. The suggested phased array feeding method requires only one laser source, an external intensity modulator, passive combiners, and dividers instead of multiple optical transmitters with multiplexers and demultiplexers.
\end{abstract}

\section{Keywords}

Phased array antennas, optical delay line, microwave photonics

\section{Introduction}

A variety of applications, including phased array antennas for radio frequency communication [1], optical communications [2], or future radar systems [3], demand the generation and control of time delays for significant frequency bandwidth microwave signals to be transmitted and processed. Delay lines are essential hardware items for applications requiring time delay as part of systems tests or evaluation.

Surface acoustic waves (SAW) based technology provides a noticeable delay at lower microwave frequencies [4]. However, the excitation and reception of the surface acoustic waves limit the behavior of this approach. The high cost and high insertion loss, particularly at higher frequencies, cannot be accepted in many applications.

On the other hand, the optical transmission also delays the transmitted radio frequency (RF) signal [5]. Fiberoptic-based microwave delay lines provide convenient RF input and output; the delay is realized between these connector points. The optical signal is intensity-modulated by the RF signal, transmitted over a fiber optic link to the receiver, and provides the proper signal delay time. The optical solution offers small size and weight, low loss and electromagnetic immunity, massive bandwidth, and performance advantages over traditional coax solutions. A continuously tunable optical delay line is a crucial element for many microwave photonics applications, including the control of optically fed phased array antennas [6], [7] or optoelectronic synchronization oscillators [8]. Hence tunability is one of the critical points of the device. The tuning accuracy of time delay is required ranging from a few picoseconds to several tens of nanoseconds.

The fiber delay-line approach is bulky and temperature-dependent. These drawbacks can be overcome by monolithic integration. Integrated photonic circuits are usually manufactured using CMOS-compatible silicon-oninsulator (SOI) technology [9]. It allows the realization of highly complex optical functionalities in a compact size and reduced unit cost. However, photonic integrated circuits usually show polarization-dependent behavior and high attenuation for unity length [10]. Also, the phase noise of the generated RF signal at the output of an integrated optoelectronic oscillator is higher than applying the fiberbased solution [11]. The optical path can be lengthened due to diminishing the light's group velocity, called slow light [12]. Many of these methods bear inherent limitations that hinder their practical development into components: they use impractical media as ultracold atomic gases, the allowed delays are modes, or they are very narrowband.

In this paper, an electronically controlled, opticalbased delay device is investigated by the theoretical description and measurements. The acquired data is used to evaluate this delay method's viability for beam steering applications in a patch antenna system. Section 2 presents the theoretical investigation of the tunable phase delay line concept. Section 3 gives measurement results on the fiberbased delay line focusing on the tunability. Section 4 presents the proposed feeding network applying the investigated fiber-based delay lines. This section also describes the simulation environment and provides system-level simulations and optimization results. Finally, the conclusion summarizes the paper. 


\section{Theoretical Investigation of the Delay Line}

The simplified block diagram of the optoelectronic delay line includes three main functional blocks. The RF signal is converted to an optical signal by a direct modulation or external electro-optical modulator in the transmitter. The intensity-modulated optical signal is transmitted over an optical waveguide. Optical fiber is used as an optical waveguide in the presented investigation, but the results can also be generalized to any integrated or non-integrated optical waveguides. The receiver converts the modulated optical signal to the electrical regime, and the delayed RF signal is realized at the output of the photodiode. As with any two-port electronic device, the RF transfer function of the delay line can be defined. The optical fiber loss is low; the electrical-optical and optical-electrical conversions dominate in the insertion loss in short transmission length. The optionally used multiplexer/combiner devices add valuable insertion loss contribution. Optical fiber has high bandwidth; the overall system transmission bandwidth depends mainly on the optical transmitter's modulation bandwidth and the receiver's detection bandwidth. The optoelectronic delay line is an active device; the laser gives some additional intensity noise; shot and thermal noises also present at the receiver's electrical output. The characteristics of the external modulator mainly limit the linearity of the transmission [13].

The delay is determined by the propagation time of the optical signal via the optical fiber. The optical length of the fiber is the physical length divided by the refractive index. Therefore, the propagation time is defined as the following:

$$
t=\frac{L \cdot n(\lambda)}{c}
$$

where $L$ is the length of the fiber, $c$ is the speed of light in vacuum, $n$ is the optical fiber refractive index, $\lambda$ is the wavelength of the optical carrier

The refractive index depends on the light wavelength through the chromatic dispersion of the optical fiber. This dependence can be calculated with the dispersion coefficient, which can be found in the fiber datasheet at a given wavelength. Near the central optical wavelength, the wavelength dependence can be modeled by linear approximation. The wavelength-dependent propagation time is defined as the following:

$$
t(\lambda) \approx \frac{L \cdot n\left(\lambda_{0}\right)}{c}+L \cdot D_{\lambda_{0}} \cdot \Delta \lambda
$$

where $D_{\lambda_{0}}$ is the dispersion coefficient at the center optical wavelength, $\Delta \lambda$ is the wavelength difference from the center wavelength.

Based on these equations, the delay time can be adjusted by the proper choice of the optical fiber, fiber length, and optical carrier wavelength.

\subsection{Length and Fiber Type-based Tunability}

The realized delay is proportional to the fiber length. Delay adjustment can be achieved with small changes in propagation distance. It is possible by stretching the fiber mechanically. For that purpose, a piezo-electric stretcher can provide electrical variation. This method is limited to typically below one ps delay variation. The temperature also affects the propagation time via two effects. The thermal expansion of the fiber tunes the physical length of the fiber with a $0.5 \cdot 10^{-6} 1 / \mathrm{K}$ thermal expansion coefficient. Also, the fiber's refractive index is changed via the thermooptic effect [14], [15]. Several applications require a substantially more significant variable time delay. The propagation length can be changed by applying different types and length fiber spools in parallel or serial setups. Electrical controlled optical switches select the proper fiber length; the optical switches' speed defines the tuning time. The number of spools defines the available length, but the resolution is limited [13].

\subsection{Dispersion-based Tunability}

Delay adjustment can also be achieved with group velocity differences in the dispersive fiber. It is proportional to the wavelength shift multiplied by the dispersive fiber's group velocity difference [16]

$$
t_{\mathrm{d}}=|D| \cdot L \cdot\left(\lambda_{1}-\lambda_{2}\right)
$$

where $t_{\mathrm{d}}$ is the delay difference, $D$ is the dispersion coefficient of the fiber, $\lambda_{1}$ is the original wavelength, and $\lambda_{2}$ is the shifted wavelength.

The group velocity is defined by the fiber's material and structural properties and the wavelength of light propagating through it. The manufacturing process determines the structural and material properties of the fiber; their tuning is practically impossible. However, the rapid, precise, and flexible control of wavelengths is feasible utilizing tunable laser sources. Since the wavelength can be tuned through laser temperature, it is possible to modify the microwave signal phase at the delay line's output by laser diode temperature control.

$$
\varphi[\mathrm{rad}]=2 \cdot \pi \cdot f \cdot t=\frac{\omega \cdot n(\lambda) \cdot L}{c}
$$

where $\varphi$ is the phase of the microwave signal at the output of the delay line, $\omega$ is the angular frequency of the transmitted microwave signal.

Applying a tunable optical source, like DWDM tunable laser with a $35 \mathrm{~nm}$ wavelength span, can realize high delay variation. If an erbium-doped fiber amplifier (EDFA) compensates for the optical loss, the wavelength is also limited by the EDFA gain bandwidth, $35 \mathrm{~nm}$ in the $\mathrm{C}$ band. The delay variation can be increased by applying CWDM lasers with $20 \mathrm{~nm}$ wavelength steps and over $200 \mathrm{~nm}$ wavelength range. In a CWDM system, optical amplifica- 
tion is impossible due to the lack of an optical amplifier with $200 \mathrm{~nm}$ amplification bandwidth.

Table 1 summarizes the parameters of the investigated fibers based on the information of the datasheets. Table 2 represents the calculated tuning range with different optical fiber types. The wavelength column shows the wavelength detuning from the initial value, while the delay column gives the differential delay achieved by this amount of detuning. The delay device introduced time delay varies linearly with the optical carrier's wavelength and the slope of the linear fit to the theoretical value computing from the dispersion coefficient. The results show that the G.652 and G.657 fibers provide the same delay tunability, but the delay's absolute value is different. However, the dispersion compensation fiber (DCF) results in higher time delay variation as a function of the wavelength due to the considerable dispersion value.

Several other optical waveguides are available, like photonic integrated solutions or plastic optical fiber. In the case of these waveguides, the applied optical wavelength, the optical attenuation, the value of the chromatics dispersion coefficient, and the behavior of its wavelength dependence are different. However, the basis of the presented operation is the same, and the method is applicable.

In several applications, the time difference is significant instead of the absolute value of the propagation time. More precisely, the phase difference of the transmitted RF signal. The time delay can be converted to phase delay (4), and the value depends on the RF signal's frequency. $1 \mathrm{ps}$ time delay causes $3.6,9,36$, and $216^{\circ}$ phase delay at $1,2.5$, 10 , and $60 \mathrm{GHz}$ RF frequency. So, 100 ps time tunability covers the whole period $\left(360^{\circ}\right)$ at $1 \mathrm{GHz}$ RF carrier. Higher tunability is unnecessary as the same time delay difference causes a higher phase delay at higher RF frequencies.

\subsection{Modeling of the Dispersion Coefficient}

The optical fiber datasheets only give a rough estimate of the chromatic dispersion coefficient at specific wavelengths. The listed zero-dispersion wavelength and dispersion slope value are not accurate enough to use in time delay calculations. The fiber dispersion must be precisely measured in the desired wavelength range (Section 3) or modeled to ensure a well controllable operation for the photonic delay device.

The previous subchapter's calculation is improved with wavelength-dependent chromatic dispersion modeling. The chromatic dispersion has two main contributions: the material dispersion coefficient increases, but the waveguide dispersion coefficient decreases versus the optical wavelength [14].

The chromatic dispersion of a standard single-mode fiber (G.652) was mathematically modeled [14] and compared with the curve's linear approximation. The chromatic dispersion coefficient is measured at $\lambda_{1}$ and $\lambda_{2}$ optical wavelength during linear approximation and assumed that the coefficient is linearly proportional with the wavelength.

\begin{tabular}{|l|c|c|}
\hline $\begin{array}{c}\text { Fiber } \\
\text { type }\end{array}$ & $\begin{array}{c}\text { Dispersion coefficient @ } \\
\mathbf{1 5 5 0} \mathbf{~ n m}[\mathbf{p s} / \mathbf{( n m} \mathbf{~ k m})]\end{array}$ & Refractive index \\
\hline $\mathrm{G} .652$ & 17 & $\begin{array}{l}1.4695 @ 1550 \mathrm{~nm} \\
1.4690 @ 1330 \mathrm{~nm}\end{array}$ \\
\hline $\mathrm{G} .657$ & 17 & $\begin{array}{l}1.4682 @ 1550 \mathrm{~nm} \\
1.4677 @ 1330 \mathrm{~nm}\end{array}$ \\
\hline $\mathrm{DCF}$ & $-100 \ldots-170(\ldots-360)$ & - \\
\hline
\end{tabular}

Tab. 1. Parameters for different fibers.

\begin{tabular}{|c|c|c|c|}
\hline \multirow{2}{*}{$\begin{array}{c}\text { Fiber } \\
\text { type }\end{array}$} & $\begin{array}{c}\text { Wavelength } \\
\text { difference } \\
{[\mathbf{n m}]}\end{array}$ & $\begin{array}{c}\text { Delay difference } \\
\text { @ 10 m fiber } \\
\text { length [ps] }\end{array}$ & $\begin{array}{c}\text { Delay difference } \mathbf{a} \\
\mathbf{1 0 0} \text { m fiber length } \\
\text { [ps] }\end{array}$ \\
\hline \multirow{2}{*}{$\mathrm{G} .652$} & 240 & 16.7 & 167 \\
\cline { 2 - 4 } & $35 @ \mathrm{C}$ band & 6 & 60 \\
\hline \multirow{2}{*}{$\mathrm{G} .657$} & 240 & 16.7 & 167 \\
\cline { 2 - 4 } & $35 @ \mathrm{C}$ band & 6 & 60 \\
\hline \multirow{2}{*}{$\mathrm{DCF}$} & 240 & 240 & 2400 \\
\cline { 2 - 4 } & $35 @ \mathrm{C}$ band & 35 & 350 \\
\hline
\end{tabular}

Tab. 2. Tunability for different fibers.

The approximated chromatic dispersion coefficient can be calculated between $\lambda_{1}$ and $\lambda_{2}$ wavelengths. The results show that less than $1 \%$ of error is expected from this linear approximation simplification inside the $35 \mathrm{~nm}$ wide C-band. This treatment gives an acceptable magnitude of error while simplifying the calculation of the expected time delay. However, the nonlinear approximation of the wavelength-dependent chromatic dispersion coefficient has to be considered by applying CWDM lasers with a large wavelength span (several times $20 \mathrm{~nm}$ wavelength differences).

\subsection{Degradation of the Behavior}

The delay device is treated as a single two-port electrical component, exhibiting a certain degree of attenuation, noise, and nonlinearity. These degradation effects have to be kept at reasonably low levels.

The noise of the optical source is characterized by the relative intensity noise (RIN). It gives information about the laser power fluctuation compared to the average output power. It is in the range of $-120 \mathrm{~dB} / \mathrm{Hz}$, and it has a maximum at the relaxation oscillation frequency in the $\mathrm{GHz}$ regime. Dark, shot and thermal noises appear at the electrical output of the photodiodes.

The nonlinearity of the Mach-Zehnder modulator must also be taken into account. High electrical-optical conversion efficiency requires high modulating RF power, further driving the modulator into its characteristic nonlinear section.

The delay's temperature dependence can be used for tunability; contrary, the operation is temperature-sensitive [14], [15]. The proper tune of the wavelength can compensate for a small fiber length difference from the theoretical length. For this purpose, a stable and fine-tunable laser source is necessary. Highly accurate, continuously tunable laser sources are available in the market with a wavelength accuracy of several pm. $10 \mathrm{pm}$ of wavelength error causes about $17 \mathrm{fs}$ positive or negative delay difference in the device equipped with $100 \mathrm{~m}$ G.652 fiber. Dispersion is a feature of the device as it opens the door to delay tunabil- 
ity. But chromatic dispersion can also degrade the quality of the transmitted RF signal [17].

\section{Experimental Investigation of the Delay Line}

\subsection{Dispersion Coefficient Measurement}

The feasibility of photonics-assisted phase shifting with a single tunable laser source was tested. The realized $\mathrm{RF}$ signal was compared with an external electrical reference. The waveforms were recorded at three different modulating frequencies $(500 \mathrm{MHz}, 1 \mathrm{GHz}, 1.5 \mathrm{GHz})$ at several laser wavelengths (fit with DWDM standard) over 1 and $10 \mathrm{~km}$ standard G.652 single-mode optical fibers. Figure 1 shows the block diagram of the measurement setup. The measured data was used to give a linear approximation of the dispersion curve in the C-band.

\subsection{Relative Phase Delay Measurement}

From the point of several applications, the tunability, namely the delay difference, is more important. For this measurement, the setup is extended by a second, fix laser source. The two optical signals were added together, modulated by one common external modulator. The modulated optical signals are transmitted via the optical fiber and separated again, converted to the electrical domain, and measured by an oscilloscope. The analog signal carried by the $1550 \mathrm{~nm}$ fix optical carrier was used as a stable trigger signal for the oscilloscope. The relative phases of the RF carriers recovered with the photodiodes at the end of the optoelectronic link were compared. It represents the relative phase shift that can be achieved by further given wavelength change at a certain fiber length and RF frequency. The measured results are summarized in Tab. 3 .

Suppose the $1 \mathrm{~km}$ long single-mode optical fiber is changed to $10 \mathrm{~km}$ long fiber, which adds about $2 \mathrm{~dB}$ of extra attenuation in the whole optical transmission. In that case, the optical power is reduced at the photodiode. Consequently, the electrical power at the output is also decreased. In the long propagation distance, the investigated RF signal is ahead of the signal carried by the optical reference carrier with 1,2 , or 4 complete cycles $(360,720$ degrees).

The expected phase is worked out based on the chromatic dispersion approximation measurement (Section 3.1). It is calculated from the fiber lengths and wavelength differences and then compared with the measured values. This gives a $0.71 \%$ phase error mean absolute value for the $1 \mathrm{~km}$ measurement, meaning that the dispersion curve acquired by measurements is valid and the phase-shifting functionality is reliable. The $10 \mathrm{~km}$ measurement error is higher because the dispersion curve was estimated based on the $1 \mathrm{~km}$ fiber spool measurement. All fiber spools were standard G.652 single-mode silica fiber, but the provider and the

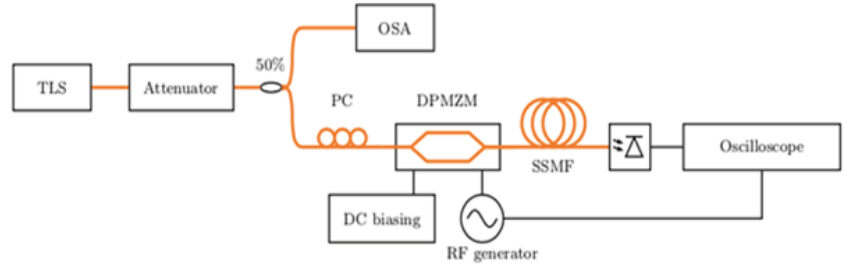

Fig. 1. Experimental setup. TLS: Tunable Laser Source, OSA: Optical Spectrum Analyzer, DPMZM: Dual Parallel Mach-Zehnder Modulator, PC: Polarization control.

\begin{tabular}{|c|c|c|c|c|}
\hline $\begin{array}{c}\text { Wavelength } \\
\text { difference } \\
{[\mathbf{n m}]}\end{array}$ & $\begin{array}{c}\text { Fiber } \\
\text { length } \\
{[\mathbf{k m}]}\end{array}$ & $\begin{array}{c}\text { RF } \\
\text { frequency } \\
{[\mathbf{G H z}]}\end{array}$ & $\begin{array}{c}\text { Measured } \\
\text { phase } \\
{\left[{ }^{\circ}\right]}\end{array}$ & $\begin{array}{c}\text { Expected } \\
\text { phase } \\
{\left[{ }^{\circ}\right]}\end{array}$ \\
\hline 3.91 & 1 & 2 & 45.01 & 45.12 \\
\hline 7.84 & 1 & 2 & 90.21 & 90.08 \\
\hline 3.91 & 10 & 2 & $80.62+360$ & $91.24+360$ \\
\hline 7.84 & 10 & 2 & $174.4+720$ & $190.84+720$ \\
\hline 3.91 & 1 & 4 & 90.42 & 90.25 \\
\hline 4.302 & 1 & 4 & 97.74 & 99.36 \\
\hline 7.84 & 1 & 4 & 180.45 & 182.17 \\
\hline 0.39 & 10 & 4 & 90.09 & 89.48 \\
\hline 3.91 & 10 & 4 & $194.7+720$ & $182.42+720$ \\
\hline
\end{tabular}

Tab. 3. Measured phase difference tuning of two RF sinusoidal signals carried by separated optical carriers over single-mode optical fiber of a given length at the given frequencies.

fabrication time were different. So, the chromatic dispersion coefficient was a little different.

\subsection{Power Level and Nonlinearity Measurement}

Another factor to consider is the eventual difference in attenuation on the paths of various wavelengths. The main contribution comes from the external modulator as a wavelength-dependent device. However, the insertion loss of the fiber and the photodiode's efficiency are also affected by the applied optical wavelength. It can be compensated using controllable attenuators or precise external modulator DC bias control. Automated bias control operation continuously can adjust the DC bias voltage of the external modulator to maintain the correct setpoint [18].

The nonlinearity of the transmission was also tested. The high RF frequency (higher than some $\mathrm{GHz}$ ) requires the application of an external modulator. The traditionally used Mach-Zehnder (MZ) and electro-absorption (EA) external modulators have worse linearity than direct modulated lasers. However, the direct modulated laser source's linearity depends on bias current, frequency, and the level of optical reflection in the system [19]. Contrary, external modulators have a more stable linearity behavior.

The measurement setup (Fig. 1) was extended by one more RF generator for two-tone measurement. The two generator's output signals are added, and the two-tones signal intensity modulates the optical carrier by applying the Mach-Zehnder modulator. IEEE linearity measurements were done at different optical carrier wavelengths. 

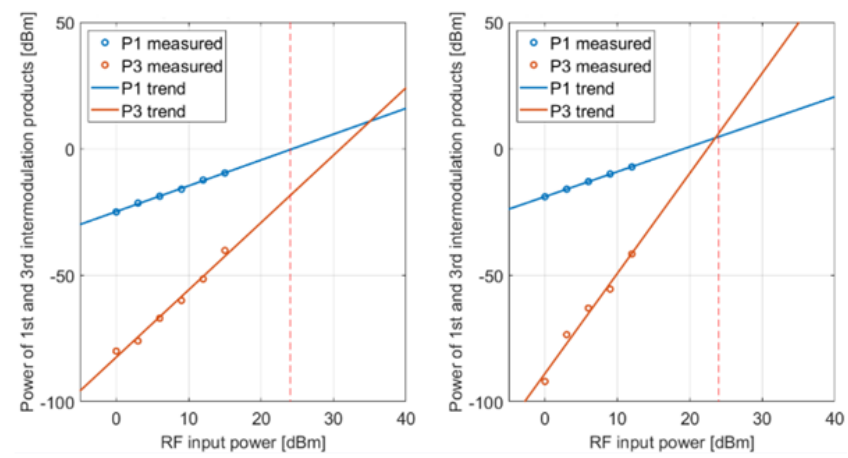

Fig. 2. Nonlinearity at two different wavelengths $(1530 \mathrm{~nm}$ and $1550 \mathrm{~nm}$ )

The power of the transmitted RF signal and the third-order intermodulation products are measured. Figure 2 shows a small difference in the transmitted RF power at the two different wavelengths. But the third-order intercept point is significantly changed. The linearity depends on the optical wavelength, mainly because the applied MZ modulator is a wavelength-dependent device, and the optimal bias point is different at the tuned optical wavelength. So, automatic bias control of the $\mathrm{MZ}$ modulator is required for proper operation [18].

\section{Antenna Beam Steering Simulation}

\subsection{Phased Array Antenna}

Phased array antenna is a key element of future mobile works [20]. It is a combination of antenna elements in which there is a control of the phase and power of the signal applied at each antenna element resulting in a wide variety of possible radiation patterns. Each antenna element requires a time delay proportional to its relative position within the array [21]. Consequently, all antenna elements are fed by the same analog electrical information but fix incremental phase differences between individual antennas [22]. If circularly polarized patch antennas are applied as array elements, the required $\Delta \varphi$ incremental phase shift between the antenna elements can be calculated to steer the radiation pattern of the array by $\theta$

$$
\Delta \varphi=\frac{360^{\circ} \cdot x \cdot \sin \theta \cdot f}{c}
$$

where $x$ is the distance of the antenna elements, $f$ is the RF carrier frequency.

\subsection{System Concept}

The proposed phased array antenna system uses a serial-feed concept realized by the electro-optical delay lines, as shown in Fig. 3. Unlike many parallel systems currently under investigation [23], [24], the proposed scheme requires one wavelength-tunable laser with one external modulator and different fiber lengths to the various antenna elements. The passive optical distribution system provides the necessary phase and true-time delays.

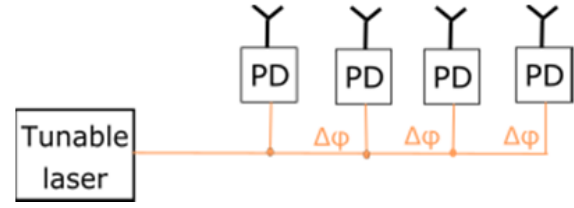

Fig. 3. Phased array antenna feed system concept.

A tunable delay line is required for beamforming application. As the laser is tuned in our design, the differential delay between fiber paths changes, and the beam is steering [13]. The proposed transmitter-phased array antenna's optical feeding system (Fig. 3) includes a tunable transmitter, optical fiber distribution system, and a photodiode at every antenna element [16]. The proportional time delay for all array elements is realized by optical wavelength control; the number of radiating directions is limited only by the number of available laser wavelengths.

\subsection{Simulation of the Radiation Pattern}

The phased array antenna is mathematically modeled as $\mathrm{N}$-slit diffraction. Each individual antenna acts as a slit, emitting radio waves; their diffraction pattern can be calculated by adding the phase shift $\varphi$ to the fringing term. The tunability of the phase shift $\varphi$ between successive antennas can adjust the angle at which the maximum energy is emitted [23], [24].

The radiation pattern simulation was realized by Matlab code. Figure 4. represents an example of the simulation results when a $1 \times 4$ subarray was fed. The phase differences between the antenna elements were $10^{\circ}, 20^{\circ}$, $40^{\circ}$, and $80^{\circ}$. The required time delay is $5.6 \mathrm{ps}, 11.2 \mathrm{ps}$, $22.4 \mathrm{ps}$, and $44.9 \mathrm{ps}$ at $5 \mathrm{GHz} \mathrm{RF}$ frequency. It can be realized by standard single-mode fiber with DWDM laser, but the required fiber length is about $50-100 \mathrm{~m}$. The fiber length can be decreased using DCF with a higher chromatic dispersion coefficient or a tunable CWDM laser source with about a $200 \mathrm{~nm}$ wavelength span. For example, CWDM
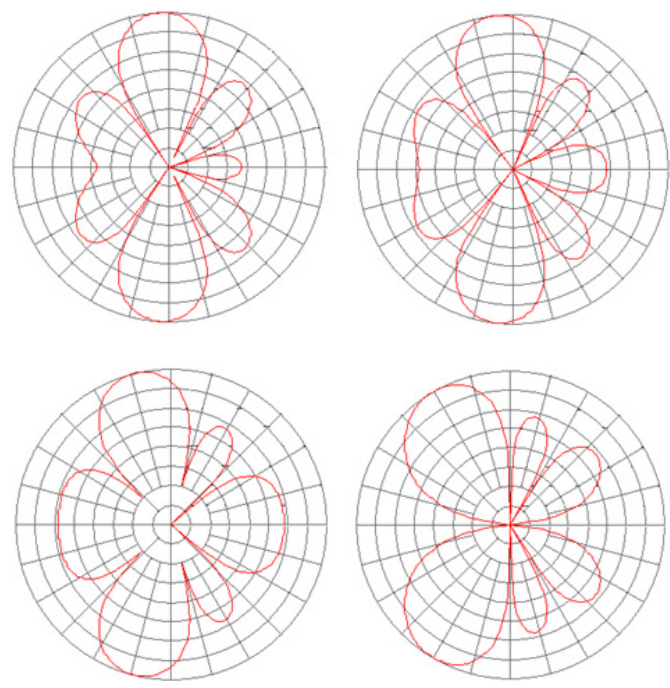

Fig. 4. Simulated radiation pattern in a polar diagram $\lambda / 2$ four-element patch antenna, $f_{\mathrm{RF}}=5 \mathrm{GHz}, \Delta \varphi=10^{\circ}$, $20^{\circ}, 40^{\circ}$, and $80^{\circ}$ 
standard tunable laser can be applied with a $20 \mathrm{~nm}$ wavelength step in the $1470-1610 \mathrm{~nm}$ range over $1.1 \mathrm{~m}$ DCF. As a validation of the operation function, the simulated farfield radiation patterns in the polar diagram (Fig. 4) are markedly modified.

A detailed antenna model is necessary to consider the feeder network's effect, the fabrication inaccuracy, and the non-ideal optoelectronic delay line operation. It was built in CST Microwave Studio environment. The simulations were made with 2 by 4 patch antenna, spaced $2 \cdot \lambda / 3$ apart, at $4 \mathrm{GHz}$ (Fig. 5).

Figure 6 shows the situation when the phase delay between the antenna elements is zero. The main lobe magnitude turned out to be $16.7 \mathrm{~dB}$, with the sidelobe level at $-14.5 \mathrm{~dB}$. The main lobe was $1^{\circ}$ away from the ideal situation and had an angular $3 \mathrm{~dB}$ width of $17.7^{\circ}$. On the right side of Fig. 6, there is still no nominal delay difference. But uniformly distributed phase error is introduced between the antenna elements, maximizing the absolute value of the error by 20 degrees. It causes a slight reduction of the main lobe magnitude with 0.1 and increases the angular width by several tenths of degrees. The sidelobe suppression is decreased by between 1 and $3 \mathrm{~dB}$, and the main lobe is steered away by less than $5^{\circ}$.

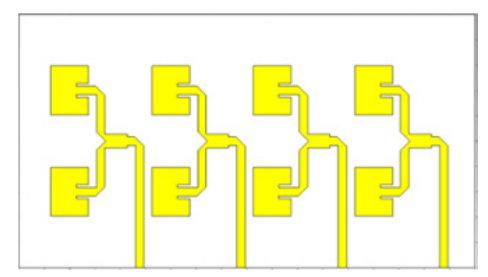

Fig. 5. Simulated $2 \times 4$ patch antenna array structure.
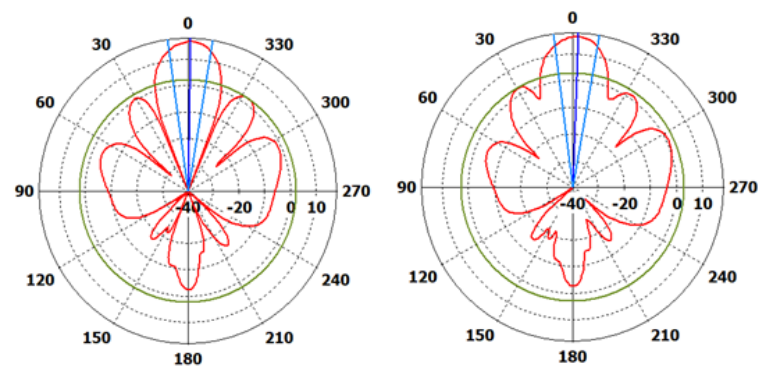

Fig. 6. Simulated radiation pattern in a polar diagram, $2 \times 4$ element patch antenna, $f_{\mathrm{RF}}=4 \mathrm{GHz}$, without phase delay, without and with error, respectively.
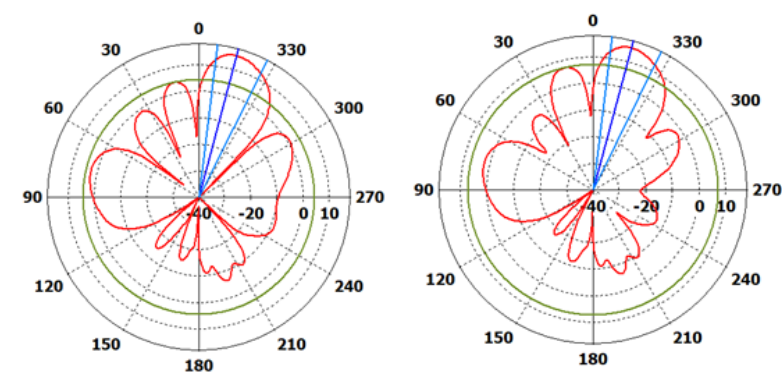

Fig. 7. Simulated radiation pattern in a polar diagram, $2 \times 4$ element patch antenna, $f_{\mathrm{RF}}=4 \mathrm{GHz}, \Delta \varphi=80^{\circ}$, without and with error, respectively.
Figure 7 shows the radiation pattern during beam steering when the delay between the antenna elements is $80^{\circ}$. Based on the simulation results, the main lobe has a magnitude of $15.3 \mathrm{~dB}$, the angular width of $19.7^{\circ}$, and the sidelobe level is $-10.9 \mathrm{~dB}$ in the ideal case. The added error has about the same effect as in the previous case; it remains in the expected domain.

The presented investigation focused on the main lobe direction and applied constant amplitude at the antenna elements. The sidelobe level can be reduced by the arrayweighting method using different attenuators in the optical branches. It is realizable, but the array-weighting optimization was not included in this paper.

\section{Conclusions}

This paper presents a method for establishing variable microwave true-time delay utilizing a photonic approach with theory, measurements, and further system simulations. The variation of the delay is achieved by applying dispersive fiber and tunable laser. The feeding network for beam steering of phased array antennas is built up based on the investigated delay line.

The theoretical investigation pointed out that the time delay for up to 360-degree phase delay difference can be realized by single-mode fiber and DWDM laser source. The chromatic dispersion coefficient's detailed model showed that $1 \%$ of error is expected from the simplification inside the $35 \mathrm{~nm}$ wide $\mathrm{C}$-band.

According to the delay measurements, the calculated delay applying linear approximation compared with the measured values gives $0.71 \%$ phase error. The experimental results also presented that the chromatic dispersion simplification has to be done based on the measurement of the same fiber. A general approximation cannot be applied for standard fiber, as the provider and the fabrication time can be different. The experimental results also showed that automatic bias control of the $\mathrm{MZ}$ modulator is required for proper operation.

The proposed phased array antenna feeding method requires just one controllable laser source and one external optical modulator. The phase difference is realized by a passive distribution network, including different fiber length branches and optical power dividers.

In the antenna system simulations, the fact was verified that the phase error introduced by the photonic delay device is still acceptable for beam steering scenarios. The added error causes a slight reduction of the main lobe magnitude with 0.1 and increases the angular width by several tenths of degrees. The sidelobe suppression is decreased by between 1 and $3 \mathrm{~dB}$, and the main lobe is steered away by less than $5^{\circ}$.

\section{Acknowledgments}

The author thanks Mr. David Kobor for his valuable contribution to the experimental work. This research was 
supported by the Ministry of Innovation and Technology and the National Research, Development and Innovation within the Quantum Information National Laboratory of Hungary.

\section{References}

[1] LI, Y., GHAFOOR, S., SATYANARAYANA, K., et al. Analogue wireless beamforming exploiting the fiber-nonlinearity of radio over fiber based C-RANS. IEEE Transactions on Vehicular Technology, 2019, vol. 68, no. 3, p. 2802-2813. DOI: 10.1109/TVT.2019.2893589

[2] DANIELSEN, S. L., MIKKELSEN, B., JOERGENSEN, C., et al. $10 \mathrm{~Gb} / \mathrm{s}$ operation of a multiwavelength buffer architecture employing a monolithically integrated all-optical interferometric Michelson wavelength converter. IEEE Photonics Technology Letters, 1996, vol. 8, no. 3, p. 434-436. DOI: 10.1109/68.481141

[3] FU, W., JIANG, D. Radar wideband digital beamforming based on time delay and phase compensation. International Journal of Electronics, 2018, vol. 105, no. 7, p. 1144-1158. DOI: 10.1080/00207217.2018.1426121

[4] BANU PRIYA, R., VENKATESAN, T., PANDYA, H. M. A comparison of surface acoustic wave (SAW) delay line modelling techniques for sensor applications. Journal of Environmental Nanotechnology, 2016, vol. 5, no. 2, p. 42-47. DOI: $10.13074 /$ jent.2016.06.162193

[5] UDVARY, E., JAKAB, L., BERCELI, T. Variable microwave time delay by utilizing optical approaches. In Proceedings of the 8th IEEE International Conference on Microwaves, Radar and Wireless Communications (MIKON). Vilnius (Lithuania), 2010, p. 1-4. INSPEC access no. 11464941

[6] ESMAN, R. D., FRANKEL, M. Y., DEXTER, J. L., et al. Fiberoptic prism true time-delay antenna feed. IEEE Photonics Technology Letters, 1993, vol. 5, no. 11, p. 1347-1349. DOI: $10.1109 / 68.250065$

[7] BOHATA, J., KOMANEC, M., SPACIL, J., et al. Experimental demonstration of a microwave photonic link using an optically phased antenna array for a millimeter-wave band. Applied Optics, 2021, vol. 60, no. 4, p. 1013-1020. DOI: 10.1364/AO.414069

[8] MIKITCHUK, K., CHIZH, A., MALYSHEV, S. Theoretical investigation of external influences on delay-line optoelectronic oscillator. In Proceedings of the International Topical Meeting on Microwave Photonics (MWP). Paphos (Cyprus), 2015, p. 1-4. DOI: 10.1109/MWP.2015.7356675

[9] SANTAGIUSTINA, M., EISENSTEIN, G., THÉVENAZ, L., et al. Slow light devices and their applications to microwaves and photonics. IEEE Photonics Society Newsletter, 2012, vol. 27, p. $5-12$.

[10] HECK, M. J. R. Highly integrated optical phased arrays: Photonic integrated circuits for optical beam shaping. Nanophotonics, 2017, vol. 6 , no. 1, p. 93-107. DOI: 10.1515/nanoph-2015-0152

[11] BOGATAJ, L., TRATNIK, J., BATAGELJ, B., et al. A highly stable OEO using a multi-purpose optical-delay stabilization system. In Proceedings of IEEE International Frequency Control Symposium (EFTF/IFCS). Besancon (France), 2017, p. 486-488. DOI: $10.1109 /$ FCS.2017.8088935

[12] BOYD, R. W., GAUTHIER, D. J., GAETA, A. L., et al Maximum time delay achievable on propagation through a slowlight medium. Physical Review A, 2005, vol. 71, no. 2, p. 1-4. DOI: 10.1103/PhysRevA.71.023801

[13] UDVARY, E., KOBOR, D., MATOLCSY, B. Photonic approaches to millimeter-wave true time-delay line. In Proceedings of the Joint Conference of the 12th International Symposium on Communication Systems, Networks and Digital
Signal Processing (CSNDSP). Porto (Portugal), 2020, p. 1-6. DOI: 10.1109/CSNDSP49049.2020.9249444

[14] ANDRE, P. S. , PINTO, A. N., PINTO, J. L. Effect of temperature on the single mode fibers chromatic dispersion. Journal of Microwaves and Optoelectronics (retrieved from Proceedings of the 2003 SBMO/IEEE MTT-S International Microwave and Optoelectronics Conference IMOC 2003, Fog do Iguazu, Brazil), 2004, vol. 3, no. 5, p. 64-70. DOI: 10.1109/IMOC.2003.1244863

[15] DING, M., FENG, Z., MARPAUNG, D., et al. Optical fiber delay lines in microwave photonics: Sensitivity to temperature and means to reduce it. Journal of Lightwave Technology, 2021, vol. 39, no. 8, p. 2311-2318. DOI: 10.1109/JLT.2021.3052609

[16] OKAWACHI, Y., SHARPING, J. E., XU, C., et al. Large tunable optical delays via self-phase modulation and dispersion. Optics Express, 2006, vol. 14, no. 25, p. 12022-12027. DOI: 10.1364/OE.14.012022

[17] HABIB, U., STEEG, M., STOHR, A., et al. Radio-over-fibersupported millimeter-wave multiuser transmission with lowcomplexity antenna units. In Proceedings of the International Topical Meeting on Microwave Photonics (MWP). Toulouse (France), 2018, p. 1-4. DOI: 10.1109/MWP.2018.8552904

[18] SHI, S., YUAN, J., HUANG, Q., et al. Bias controller of MachZehnder modulator for electro-optic analog-to-digital converter. Micromachines, 2019, vol. 10, no. 12, p. 1-10. DOI: 10.3390/mi10120800

[19] MAROZSAK, T., UDVARY, E., KOVACS, A., et al. Effect of optical reflection on nonlinear characteristics of direct modulated lasers. In Proceedings of the International Topical Meeting on Microwave Photonics (MWP). Budapest (Hungary), 2003, p. 227-230. DOI: 10.1109/MWP.2003.1422873

[20] HUSSAIN, B. SERAFINO, G., AMATO, F., et al. Fast photonicsassisted beamforming network for wide-band, high bit rate $5 \mathrm{G}$ communications. In Proceedings of the International Topical Meeting on Microwave Photonics (MWP). Toulouse (France), 2018, p. 1-4. DOI: 10.1109/MWP.2018.8552884

[21] SERAFInO, G., PORZI, C., FALCONI, F., et al. Photonicsassisted beamforming for $5 \mathrm{G}$ communications. IEEE Photonics Technology Letters, 2018, vol. 30, no. 21, p. 1826-1829. DOI: 10.1109/LPT.2018.2874468

[22] HABIB, U., STEEG, M., STOHR, A., et al. Single radio-overfiber link and RF chain-based $60 \mathrm{GHz}$ multi-beam transmission. IEEE Journal of Lightwave Technology, 2019, vol. 37. no. 9, p. 1974-1980. DOI: 10.1109/JLT.2019.2896778

[23] RITOŠA, P., BATAGELJ, B., VIDMAR, M. Optically steerable antenna array for radio over fibre transmission. IET Electronics Letters, 2005, vol. 41, no. 16, p. 917-918. DOI: 10.1049/el:20051308

[24] AKIBA, S., OISHI, M., NISHIKAWA, Y., et al. Photonic approach to beam steering of phased array antenna. In Proceedings of the International Symposium on Electromagnetic Theory. Hiroshima (Japan), 2013, p. 448-451. INSPEC accession no.: 13682435

\section{About the Author ...}

Eszter UDVARY received a Ph.D. degree in Electrical Engineering from Budapest University of Technology and Economics (BME), Budapest, Hungary, in 2009. She is currently an Associate Professor at BME, Mobile Communication and Quantum Technologies Laboratory. Dr. Udvary's research interests are in the broad area of optical communications, include microwave photonics, optical access network, visible light communication, and quantum communication. 